\title{
Weighted trace formula near a hyperbolic trajectory and complex orbits
}

\author{
T. Paul ${ }^{\mathrm{a})}$ \\ CEREMADE, URA 749 CNRS, Université Paris-Dauphine, Place de Lattre de Tassigny, \\ 75775, Paris, Cedex 16, France
}

A. Uribe

Department of Mathematics, University of Michigan, Ann Arbor, Michigan 48109

(Received 15 January 1998; accepted for publication 3 April 1998)

In this paper we consider a weighted trace formula for Schrödinger operators. More precisely, let $\psi_{j}^{\hbar}$ and $E_{j}^{\hbar}$ denote the eigenfunctions and eigenvalues of a Schrödinger-type operator $H_{\hbar}$ with a discrete spectrum. Let $\psi_{(x, \xi)}$ be a coherent state centered at a point $(x, \xi)$ of a hyperbolic closed orbit $\gamma$. We show that, as $\hbar \rightarrow 0$, the leading term of $\sum_{j} \varphi\left\{\left[E_{j}(\hbar)-E\right] / \hbar\right\}\left|\left(\psi_{(x, \xi)}, \psi_{j}^{\hbar}\right)\right|^{2}$ can be expressed in terms of the analytic continuation on the upper and lower half-planes of the positive and negative frequencies part of $\varphi$. The result is also related to complex trajectories surrounding $\gamma$. (C) 1998 American Institute of Physics. [S0022-2488(98)01908-2]

\section{INTRODUCTION}

Consider a Schrödinger operator $H=-\hbar^{2} \Delta+V(x)$ with $V$ smooth, on $\mathbb{R}^{n}$ (in which case we assume $V$ tends to infinity at infinity and therefore $H$ has a discrete spectrum) or on a compact Riemannian manifold, $M$.

The trace (Gutzwiller) formula (Ref. 1) expresses the smeared out spectral density over a Fourier compactly supported test function: let $\left\{E_{j}\right\}$ and $\left\{\varphi_{j}\right\}$ be the eigenvalues and eigenfunctions of $H$. Then, under certain hypothesis on the classical flow (for example, that the periodic trajectories of energy $E$ are isolated),

$$
\sum_{j} \varphi\left(\frac{E_{j}-E}{\hbar}\right) \sim \sum_{k=0}^{\infty} c_{k}(\varphi) \hbar^{-n+1+k}+\sum_{\gamma} \sum_{l=0}^{\infty} d_{\gamma}^{l}(\varphi) \hbar^{l}
$$

where $c_{k}$ are distributions whose Fourier transform are supported on 0 , the second sum is over the periodic trajectories $\gamma$ of energy $E$, and $d_{\gamma}^{l}$ have Fourier transforms supported on the set of periods of $\gamma$.

We remarked in Ref. 2 that one can isolate the contribution of a given periodic trajectory by ponderating the sum (1) by the so-called Husimi function of $\varphi_{j}$ : let $\psi_{(x, \xi)}^{a}$ be a coherent state at $(x, \xi) \in \mathbb{R}^{2 n}$ of "vacuum" $a \in \mathscr{P}\left(\mathbb{R}^{n}\right)$ :

$$
\psi_{(x, \xi)}^{a}(y)=\rho(y-x)(2 \pi \hbar)^{-3 n / 4} 2^{-n / 4} e^{-i x \xi / 2 \hbar} e^{i \xi y / \hbar} a\left(\frac{y-x}{\sqrt{\hbar}}\right)
$$

(here $\rho$ is a compactly supported $C^{\infty}$ function equal to 1 near zero). Let $(x, \xi) \in \gamma$, where $\gamma$ is a periodic trajectory of the classical underlying flow of energy $E:=\xi^{2}+V(x)$. We showed that

$$
\sum \varphi\left(\frac{E_{j}-E}{\hbar}\right)\left|\left(\psi_{(x, \xi)}^{a}, \varphi_{j}\right)\right|^{2} \sim \sum_{k=0}^{\infty} \nu_{k}(\varphi) \hbar^{-n+1 / 2+k}
$$

where the $\nu_{k}$ are distributions whose Fourier transforms are supported on the set of periods of (iterates of) $\gamma$.

${ }^{a)}$ Electronic mail: paulth@zin.ceremade.dauphine.fr 
Let us suppose for simplicity that the dimension $n=2$ (the results being immediately extended to the general case). We remarked in Ref. 2 that if $\gamma$ is elliptic, i.e., if the Poincare mapping of $\gamma$ is a rotation of angle $\theta$, the distribution $\nu_{0}$ could be expressed as a Dirac measure on the energies of the quasimodes associated to $\gamma$ :

$$
\nu_{0}(\varphi)=\sum_{j, k \in Z} c_{k} \varphi\left(\frac{1}{T_{\gamma}}\left(2 \pi j+\left(k+\frac{1}{2}\right) \theta+\frac{S_{\gamma}}{\hbar}+\sigma_{\gamma}\right)\right)
$$

(here $T_{\gamma}, S_{\gamma}$, and $\sigma_{\gamma}$ are the period, action, and Maslov index of $\gamma$ ). On the contrary, if $\gamma$ is hyperbolic the measure $\nu_{0}$ is Lebesgue continuous.

In this paper we want to give another formulation of $\nu_{0}$ that involves the complex trajectories of the linearized near $\gamma$, i.e., the complex trajectories infinitesimally close to $\gamma$ (see Theorem 2.1 below). Before we state the results we would like to comment on the role of complex periodic trajectories in the study of the semiclassical spectrum of Schrödinger operators.

Usually the complex trajectories appear to give exponential corrections to the semiclassical expansions. Among the works dedicated to this problem let us mention the work of Balian and Bloch on the trace formula $;{ }^{3}$ the resurgence method of Ecalle and Voros; ${ }^{4-6}$ and more recently, the work by Fefferman and Secco $^{7}$ on the correction term of the number of negative eigenvalues of atomic systems.

More recently, Delande et al. ${ }^{8}$ and Eckhardt et al., ${ }^{9}$ discussed the trace formula for a mapping depending of a parameter near a bifurcation.

A formal computation of the contribution of a given hyperbolic trajectory to the trace formula was given in Ref. 10, where it was remarked, however, that the contribution of different periodic trajectories should interfere destructively. The use of coherent states in the present paper will isolate a given trajectory by microlocalizing near a point in phase space. This makes only one trajectory contributing and the complexified linearized flow near this trajectory present already at the leading order.

The paper is organized as follows: the results are presented in the next section and proven in Sec. III. Section IV contains some concluding remarks.

\section{THE RESULTS}

Let $H$ be as in the Introduction. Let $\gamma$ be a hyperbolic closed trajectory of the Hamiltonian $\xi^{2}+V(x)$. Let $\mu$ be the Lyapounov exponent at $(x, \xi) \in \gamma$ [that is, let $e^{ \pm \mu}$ be the two eigenvalues of the Poincaré mapping of $\gamma$ at $(x, \xi)]$ and $\varphi \in \mathscr{S}(\mathbb{R})$ be such that its Fourier transform $\hat{\varphi}$ is compactly supported. We will denote $\varphi^{ \pm}$the Hardy and anti-Hardy part of $\varphi$, namely,

$$
\varphi^{ \pm}(x):=\int_{\mathbb{R}^{ \pm}} \hat{\varphi}(\xi) e^{-i \xi x} d \xi
$$

We will denote $W_{a}$ the Wigner function of the symbol $a$ (see the next section) and express $W_{a}$ on the variables $\left(x_{T}, x_{\perp}, x_{s}, x_{u}\right)$, where $x_{T}$ is tangent to $\gamma, x_{s}$ and $x_{u}$ are along the stable and unstable manifold of the Poincare mapping, and $x_{\perp}$ is a transverse direction to the energy shell.

Then we have the following.

Theorem 2.1: Let us suppose that

$$
\sum_{k=0}^{\infty}\left(2 \frac{1-e^{-\mu}}{1+e^{-\mu}}\right)^{k} \int\left|W_{a}\left(x_{T}, x_{\perp}=0, x_{s}, x_{u}\right)\right| \frac{\left|x_{s} x_{u}\right|^{k}}{k !} d x_{T} d x_{s} d x_{u} \leqslant \infty
$$

Then as $\hbar \rightarrow 0$ along any sequence of the type

$$
\begin{gathered}
\hbar=\frac{S_{\gamma}}{2 \pi k+\sigma_{\gamma}+\alpha}, \quad \alpha \in[0,1[, \quad k \rightarrow \infty, \\
\sum_{j} \varphi\left(\frac{E_{j}(\hbar)-E}{\hbar}\right)\left|\left(\psi_{(x, \xi)}^{a}, \psi_{j}^{\hbar}\right)\right|^{2}=\hbar^{-n+1 / 2} \sum_{l, m \in \mathbb{Z}} c_{l m} \varphi^{\operatorname{sign}(m)}\left(z_{l m}\right)+O\left(\hbar^{-n+3 / 2}\right),
\end{gathered}
$$


where $z_{l m}:=\left(2 \pi / T_{\gamma}\right)\left(l+i\left(m+\frac{1}{2}\right) \mu+\alpha\right), l, m \in \mathbb{Z}$. Moreover,

if $m \geqslant 0$,

$$
c_{l m}=\frac{1}{m !} \int x_{u}^{m} \partial_{x_{s}}^{m} W^{a}\left(x_{T}, x_{\perp}=0, x_{s}, x_{u}\right) e^{i x_{s} x_{u}} d x_{T} d x_{s} d x_{u} ;
$$

if $m<o$,

$$
c_{l m}=\frac{1}{m !} \int x_{s}^{m} \partial_{x_{u}}^{m} W^{a}\left(x_{T}, x_{\perp}=0, x_{s}, x_{u}\right) e^{i x_{s} x_{u}} d x_{T} d x_{s} d x_{u} .
$$

Remark: The hypothesis (6) is a condition of concentration in phase space of the symbol $a$ near $\gamma$ along the stable and unstable manifolds. An example of a symbol satisfying (6) is any Gaussian [since in this case the Wigner function is itself a Gaussian and so satisfies (6), as an easy computation shows].

The next result gives a dynamical interpretation of the numbers $z_{l m}$. In the elliptic case, namely when the linearized flow near $\gamma$ is stable, one can think, as in Ref. 11 (see also Refs. 12 and 13), at the flow around $\gamma$ as being integrable, namely, as seating on an (infinitesimal) torus around $\gamma$. There are then two "actions" arising: the one parametrizing the continuous family of periodic trajectories that $\gamma$ belongs to (see Ref. 2), and the one coming from the Poincare mapping. This leads to the following normal form:

$$
E(A, B)=E_{c}(A)+\frac{\theta}{T_{\gamma}} B, \quad B \text { small, } A \text { near } S_{\gamma},
$$

with

$$
\frac{\partial E}{\partial A}\left(S_{\gamma}, B\right)=\frac{2 \pi}{T_{\gamma}} .
$$

Indeed, $E(A, B)$ induces on the angles $\varphi_{A}, \varphi_{B}$, conjugate to $A, B$ a flow at time $T_{\gamma}$ given by:

$$
\begin{gathered}
\varphi_{A}\left(T_{\gamma}\right)=\varphi_{A}(0)+2 \pi, \\
\varphi_{B}\left(T_{\gamma}\right)=\varphi_{B}(0)+\theta .
\end{gathered}
$$

The numbers $\left(1 / T_{\gamma}\right)\left(2 \pi j+\left(k+\frac{1}{2}\right) \theta+\left(S_{\gamma} / \hbar\right)+\sigma_{\gamma}\right)$ appearing in (4) are precisely the quantities $\left[E\left(A_{j}, B_{k}\right)-E\right] / \hbar$, where $A_{j}$ and $B_{k}$ are the values of the actions quantized by Bohr-Sommerfeld conditions:

$$
A_{j}=S_{\gamma}+\left(j+\sigma_{\gamma}\right) \hbar \quad \text { and } \quad B_{k}=\left(k+\frac{1}{2}\right) \hbar .
$$

We want to show that this situation is still valid in the hyperbolic case if we consider complex torus and complex actions.

Let $P_{\gamma}$ be the Poincare mapping of $\gamma$. Since $\gamma$ is hyperbolic $P_{\gamma}$ can be represented as a matrix of the form

$$
P_{\gamma}=\left(\begin{array}{cc}
\cosh \mu & \sinh \mu \\
\sinh \mu & \cosh \mu
\end{array}\right) .
$$

We will extend this mapping to the complex in the following way: consider the complex symplectic dilation $D_{\sqrt{i}}$ in $\mathrm{R}^{2} \otimes \mathrm{C}$ :

$$
\left(\begin{array}{l}
x \\
\xi
\end{array}\right)^{D \sqrt{i}} \rightarrow\left(\begin{array}{c}
\sqrt{i x} \\
\frac{1}{\sqrt{i}} \xi
\end{array}\right):=\left(\begin{array}{l}
z_{1} \\
z_{2}
\end{array}\right) .
$$


Under this transformation, the mapping $P_{\gamma}$ becomes

$$
D_{\sqrt{i}}^{-1} P_{\gamma} D_{\sqrt{i}}=\left(\begin{array}{cc}
\cosh (i \mu) & -i \sinh (i \mu) \\
i \sinh (i \mu) & \cosh (i \mu)
\end{array}\right)=\left(\begin{array}{cc}
\cos (i \mu) & -\sin (i \mu) \\
\sin (i \mu) & \cos (i \mu)
\end{array}\right)=R(i \mu)
$$

where $R(\theta)$ is the rotation of angle $\theta$.

This means that, thanks to this symplectic complex dilation, we are back to an "elliptic", situation with complex time. In particular, we find that there exist complex stable tori and a complex normal form $F(A, B)$ satisfying

$$
F(A, B)=E_{c}(A)+i \frac{\mu}{T_{\gamma}} B
$$

which gives rise to quantized Bohr-Sommerfeld values of the energy $F\left(A_{j}, B_{k}\right)$ with $A_{j}$ and $B_{k}$ given by (13).

We just proved the following.

Proposition 2.2: Let $z_{l m}$ as in Theorem 2.1; then

$$
z_{l m}=F\left(A_{l}, B_{m}\right)
$$

with

$$
A_{j}=S_{\gamma}+\left(j+\sigma_{\gamma}\right) \hbar \text { and } B_{k}=\left(k+\frac{1}{2}\right) \hbar .
$$

We will come back to the interpretation of this result in the final section of the paper.

\section{PROOF OF THE THEOREM}

In Ref. 2 we proved that the first coefficient $\nu_{0}(\varphi)$ in (3) can be written as

$$
\nu_{0}(\varphi)=\sum_{n} e^{i n\left[\left(S_{\gamma} / \hbar\right)+\sigma_{\gamma}\right]} \int_{-\infty}^{\infty}\left(a, Z(s(\dot{x}, \dot{\xi})) U^{n} a\right) d s \hat{\varphi}\left(n T_{\gamma}\right) ;
$$

here $(\dot{x}, \dot{\xi})$ is the tangent vector to the flow at $(x, \xi) ; Z$ is a Weyl operator defined by

$$
Z(e, f) a(\eta)=e^{-i(e f / 2)} e^{i e \eta} a(\eta-f),
$$

and $U$ is the metaplectic operator of the linearized low at time $T_{\gamma}$ (see Ref. 2).

We also showed that one can find a symplectic mapping $R$ such that $U=M\left(S\left(T_{\gamma}\right)\right)$, where $M$ is the metaplectic representation such that

$$
R^{-1} S\left(T_{\gamma}\right) R=\left(\begin{array}{cccc}
1 & 0 & \alpha & 0 \\
0 & e^{-\mu} & 0 & 0 \\
0 & 0 & 1 & 0 \\
0 & 0 & 0 & e^{\mu}
\end{array}\right)
$$

where $\alpha \in \mathbb{R}$ and $\mu$ is the local Lyapounov exponent of $\gamma$ at $(x, \xi)$.

Let us denote $a^{\prime}:=M(R) a$. Then one easily checks that, if $\hbar$ satisfies (6),

$$
\begin{aligned}
& \nu_{0}(\varphi)=\sum_{n} e^{i n \alpha} \int \overline{a^{\prime}(\eta)} e^{i n(\alpha / 2) \partial^{2}} \eta_{1} e^{-n(\mu / 2)} a^{\prime}\left(\eta_{1}-s, e^{-n \mu} \eta_{2}\right) d \eta d s \\
& =\sum_{n} e^{-n(\mu / 2)} e^{i n \alpha} \int \overline{a^{\prime}\left(0, \eta_{2}\right)} a^{\prime}\left(0, e^{-\mu} \eta_{2}\right) d \eta_{2},
\end{aligned}
$$

where $a^{\prime}$ is the Fourier transform of $a^{\prime}$ with respect to $\eta_{1}$. Let us call $b(x):=a^{\prime}(0, x)$ and let $W_{b}(x, \xi)$ be the Wigner function of $b$, namely, 


$$
W_{b}(x, \xi):=\int \overline{b(x-y)} b(x+y) e^{2 i \xi y} d y
$$

Lemma 3.1: Let us suppose that

$$
\sum_{k=0}^{\infty}\left(2 \frac{1-e^{-\mu}}{1+e^{-\mu}}\right)^{k} \int\left|W_{b}(x, \xi)\right| \frac{|x \xi|^{k}}{k !} d x d \xi \leqslant \infty
$$

then

$$
e^{-n(\mu / 2)} \int \overline{a^{\prime}\left(0, \eta_{2}\right)} a^{\prime}\left(0, e^{-\mu} \eta_{2}\right) d \eta_{2}=\sum_{k=0}^{\infty} \frac{\overline{b^{(k)}(0)} \hat{b}^{(k)}(0)}{k !} e^{-(k+1 / 2) \mu}
$$

where $b^{(k)}$ is the kth derivative of $b$.

Proof: We have, by straightforward computations,

$$
\begin{aligned}
\int \overline{a^{\prime}\left(0, \eta_{2}\right)} a^{\prime}\left(0, e^{-\mu} \eta_{2}\right) d \eta_{2} & =\int \overline{b(x)} b\left(e^{-\mu} x\right) d x \\
& =\int W_{b}\left(\frac{x\left(1+e^{-\mu}\right)}{2}, \xi\right) e^{-i \xi x\left(1-e^{-\mu}\right)} d x d \xi \\
& =\frac{2}{1+e^{-\mu}} \int W_{b}(x, \xi) e^{-2 i \xi x\left[\left(1-e^{\left.\left.-\mu / 1+e^{-\mu}\right)\right]} d x d \xi\right.\right.}
\end{aligned}
$$

(25) implies that the RHS of (27) is convergent. Therefore (26) is also convergent.

Let us show now that the Lemma implies the formula (8). Since $\hat{\varphi}$ is compactly supported we can plug (20) in the expansion (26) and invert the summations. It now suffices to note that $e^{(-\xi)} \chi_{[0,+\infty[}$ is the Fourier transform of $2 \pi /(i-x)$ and use the Cauchy and Poisson formulas to get

$$
\nu_{0}(\varphi)=\sum_{l, m \in \mathbb{Z}} c_{l m} \varphi^{\operatorname{sign}(m)}\left(z_{l m}\right)
$$

with

$$
c_{l m}=\frac{\overline{b^{(k)}(0)} \hat{b}^{(k)}(0)}{k !} \text {. }
$$

We need now to express the hypothesis (25) and the $c_{l m}$ in terms of the Wigner function of $a$. To do so let us first remark that if $S$ is a symplectic mapping and $M$ the metaplectic representation, we have

$$
W_{M(S) a}(x, \xi)=\left(W_{a} o S\right)(x, \xi)
$$

Together with the fact that

$$
\overline{\hat{a}(0)} \hat{a}(0)=\int W_{a}(0, \xi) d \xi
$$

we get easily that (25) is equivalent to

$$
\sum_{k=0}^{\infty}\left(2 \frac{1-e^{-\mu}}{1+e^{-\mu}}\right)^{k} \int\left|W_{a}\left(x_{T}, x_{\perp}=0, x_{s}, x_{u}\right)\right| \frac{\left|x_{s} x_{u}\right|^{k}}{k !} d x_{T} d x_{s} d x_{u} \leqslant \infty
$$

and the expression (9) and (10) for the coefficients. 


\section{COMMENTS}

Link with "top resonances": although the operator $H$ is elliptic with discrete spectrum, which implies that there are no "resonances," the formula (8) suggests that the Hardy and anti-Hardy parts of the weighted spectral density $\rho(\lambda):=\sum \delta\left(\lambda-\left[E_{j}(\hbar)-E\right] / \hbar\right)\left|\left(\psi_{(x, \xi)}^{a}, \psi_{j}^{\hbar}\right)\right|^{2}$ have poles, in the semiclassical limit. These poles are precisely located on the same lattice that in the case of the so-called "top resonances" (Refs. 14-16): if the potential $V$ tends to zero at infinity and if there is an unstable fixed point or a manifold of unstable fixed points on the energy surface, then one can prove that $H$ has resonances at a distance $\sim \hbar$ from the real axis. Our result gives a microlocalized version of this phenomenon.

Link with the "analytic dilation" method: let us look at the poles $z_{l 0}$ and the corresponding coefficient $c_{0}$,

$$
c_{l 0}=\int W^{a}\left(x_{T}, x_{\perp}=0, x_{s}, x_{u}\right) e^{i x_{s} x_{u}} d x_{T} d x_{s} d x_{u} .
$$

Calling

$$
\left(\begin{array}{l}
x \\
\xi
\end{array}\right):=\left(\begin{array}{c}
\frac{x_{s}+x_{u}}{\sqrt{2}} \\
\frac{x_{s}-x_{u}}{\sqrt{2}}
\end{array}\right),
$$

one gets

$$
c_{l 0}=\int W^{a}\left(x_{T}, x_{\perp}=0, x, \xi\right) e^{i\left[\left(\xi^{2}-x^{2}\right) / 2\right]} d x_{T} d x d \xi
$$

Using elementary properties of the Wigner function, one gets that

$$
c_{l 0}=\left(b, g^{+}\right)\left(g^{-}, b\right),
$$

where $b$ was defined in Sec. III and $g^{ \pm}(x):=e^{ \pm i\left(x^{2} / 2\right)}$.

Moreover, the same computation in the case of $\gamma$ elliptic gives rise to

$$
c_{l 0}^{e l l}=(b, g)(g, b),
$$

where $g(x):=e^{-x^{2} / 2}$. Let $|g\rangle\langle g|$ be the orthogonal projector on the vector $g$ in $L^{2}(\mathbb{R})$. If one calls $D_{\alpha}$ the operator of dilation by $\alpha$, one sees that $\left|g^{+}\right\rangle\left\langle g^{-}\right|$is the analytic continuation of $D_{\alpha}^{-1}|g\rangle\langle g| D_{\alpha}$ evaluated at $\alpha=\sqrt{i}$, and so is $c_{l 0}:=\left(b,\left|g^{+}\right\rangle\left\langle g^{-}\right| b\right)$. This suggest that the poles of the weighted spectral measure can be obtained, as the usual resonances do, by analytic dilation.

Link with "normal forms": in Refs. 17 and 18, Guillemin introduced quantized normal forms near a closed trajectory. The result of this paper suggests that the complex dilation of this normal form gives rise to poles of the spectral density suitably microlocalized.

\section{ACKNOWLEDGMENT}

The research of A. Uribe was supported, in part, by National Science Foundation Grant No. DMS-9107600.

${ }^{1}$ T. Paul and A. Uribe, "The semi-classical trace formula and propagation of wave packets," J. Funct. Anal. 132, 192-249 (1995).

${ }^{2}$ T. Paul and A. Uribe, "On the pointwise behaviour of semi-classical measures," Commun. Math. Phys. 175, 229-258 (1996).

${ }^{3}$ R. Balian and C. Bloch, Ann. Phys. (N.Y.) 63, 592-606 (1971); 64, 271-307 (1971); 69, 76-160 (1972); 85, 514-545 (1974).

${ }^{4}$ J. Ecalle, "Les fonctions résurgentes," Publications Mathématiques de l'Université Paris Sud, 81-05 and 81-06, 1981.

${ }^{5}$ A. Voros, "The return of the quartic oscillator. The complex WKB method," Ann. Inst. Henri Poincaré, Sect. A 34, 211-388 (1983). 
${ }^{6}$ A. Voros and P. Cartier, in Grothendieck Festschrift (Birkhauser, Basel, 1990), Vol. 2.

${ }^{7}$ A. Córdoba, C. Feffermann, and L. Secco, Weyl Sums and Atomic Energy Oscillations Revista Matemática Iberoamericana (1995), Vol 11, No. 1. pp. 167-228.

${ }^{8}$ D. Delande, M. Kus, and F. Haake, "Prebifurcation periodic ghost orbits in semi-classical quantization," Phys. Rev. Lett. 71, 2167-2171 (1993).

${ }^{9}$ B. Eckhardt, M. Kus, and F. Haake, Z. Phys. B 65, 221 (1993).

${ }^{10}$ A. Voros, "Unstable periodic orbits and semi-classical quantization," J. Phys. A 21, 685-692 (1988).

${ }^{11}$ A. Voros, "The WKB-Maslov method for nonseparable systems Coll. Inst. CNRS n. 237," Géométrie Symplectique et Physique Mathématique, pp. 217-252.

${ }^{12}$ J. V. Ralston, "On the construction of quasimodes associated with stable periodic orbits," Commun. Math. Phys. 51, 219-242 (1976).

${ }^{13}$ Y. Colin de Verdière, “Quasi-modes sur les varietes Riemanniennes,” Invent. Math. 43, 15-42 (1977).

${ }^{14}$ T. Paul, Thèse d'etat, Université d'Aix-Marseille, 1985.

${ }^{15}$ R. Briet, P. Duclos, and J. M. Combes, Commun. Part. Diff. Eq. 12, 201 (1987).

${ }^{16}$ C. Gérard and J. Sjöstrand, Commun. Math. Phys. 108, 391-421 (1987).

${ }^{17}$ V. Guillemin, "Wave-trace invariants and a theorem of Zelditch," Bull. Am. Math. Soc. 12, 303-308 (1993).

${ }^{18}$ V. Guillemin, "Wave-trace invariants," Duke Math. J. 83, 287-352 (1996). 